\title{
The utility of 3D printing for surgical planning and patient-specific implant design for complex spinal pathologies: case report
}

\author{
Ralph J. Mobbs, MD, FRACS, ${ }^{1-3}$ Marc Coughlan, MBBS, FRACS, ${ }^{2}$ \\ Robert Thompson, MBus, BInfoTech, DipAppSci, ${ }^{4}$ Chester E. Sutterlin III, MD, ${ }^{5-7}$ and \\ Kevin Phan, BSc ${ }^{1-3}$
}

${ }^{1}$ NeuroSpine Surgery Research Group (NSURG), Prince of Wales Private Hospital, Sydney; ${ }^{2}$ Prince of Wales Private Hospital, Sydney; ${ }^{3}$ University of New South Wales, Sydney; ${ }^{4}$ Anatomics Pty. Ltd., Melbourne, Australia; and ${ }^{5}$ Department of Neurosurgery, University of Florida, Gainesville; ${ }^{6}$ Spinal Health International, Inc., Longboat Key, Florida; and PProCRO Pty. Ltd., Sydney, New South Wales, Australia

OBJECTIVE There has been a recent renewed interest in the use and potential applications of 3D printing in the assistance of surgical planning and the development of personalized prostheses. There have been few reports on the use of $3 \mathrm{D}$ printing for implants designed to be used in complex spinal surgery.

METHODS The authors report 2 cases in which 3D printing was used for surgical planning as a preoperative mold, and for a custom-designed titanium prosthesis: one patient with a C-1/C-2 chordoma who underwent tumor resection and vertebral reconstruction, and another patient with a custom-designed titanium anterior fusion cage for an unusual congenital spinal deformity.

RESULTS In both presented cases, the custom-designed and custom-built implants were easily slotted into position, which facilitated the surgery and shortened the procedure time, avoiding further complex reconstruction such as harvesting rib or fibular grafts and fashioning these grafts intraoperatively to fit the defect. Radiological follow-up for both cases demonstrated successful fusion at 9 and 12 months, respectively.

CONCLUSIONS These cases demonstrate the feasibility of the use of 3D modeling and printing to develop personalized prostheses and can ease the difficulty of complex spinal surgery. Possible future directions of research include the combination of 3D-printed implants and biologics, as well as the development of bioceramic composites and custom implants for load-bearing purposes.

https://thejns.org/doi/abs/10.3171/2016.9.SPINE16371

KEY WORDS 3D printing; model; surgery; simulation; implant; design; chordoma; lumbar fusion; cervical

$\mathrm{T}$ HE reconstruction of the spine and skull has a rich and longstanding history, spanning at least 5 millennia to include attempts in which a range of materials has been used, from fruit shells, to sheep scapulae, to man-made plastics. ${ }^{3,14}$ There has been a recent renewed interest in the use and potential applications of 3D printing in these realms, as a source of customizable patient-specific implants for the nuances of each patient's individual anatomy. Medical applications for 3D printing are expanding at a rapid pace and are expected to revolutionize health care. ${ }^{12}$ Currently there is innovative research into the appli- cations of 3D printing in several avenues, including manufacturing of anatomical models, ${ }^{4,6}$ customized prosthetics and implants, and tissue and organ fabrication, as well as drug delivery vehicles and discovery.

There have been reports of the application of 3D printing for preoperative surgical planning: ${ }^{4,6}$ however, there have been few reports on implant design for complex spinal surgery. With computer 3D modeling software and the patient's neuroimaging data, a mold of the patient's anatomy as well as a corresponding patient-specific implant can be fabricated. We report 2 cases in which $3 \mathrm{D}$ printing was

ABBREVIATIONS CAD = computer-aided design; C.S.I.R.O. = Commonwealth Scientific and Industrial Research Organisation; Oc = occiput.

SUBMITTED March 29, 2016. ACCEPTED September 13, 2016.

INCLUDE WHEN CITING Published online January 20, 2017; DOI: 10.3171/2016.9.SPINE16371. 
used for surgical planning as a preoperative mold, and for a custom-designed titanium prosthesis: one patient with a C-1/C-2 chordoma who underwent tumor resection and vertebral reconstruction, and another patient with a custom-designed titanium anterior fusion cage for an unusual congenital spinal deformity.

\section{Case Reports \\ Case 1}

A 63-year-old man presented to our institution with a 3 -month history of neck and shoulder pain. The cervical CT and MRI studies demonstrated an osteolytic lesion involving the $\mathrm{C}-2$ vertebral body and the anterior $\mathrm{C}-1$ arch (Fig. 1). The lesion occupied these 2 vertebrae without lateral extent, or growth into the vertebral foramen, but extended up to the anterior epidural space at C-2. A CTguided transoral biopsy revealed chordoma.

Tumor removals at the craniocervical junction have historically been challenging and highly morbidity-producing procedures. There have been only limited attempts at tumor resection and structural reconstruction in the area below the occiput (Oc), given the narrow operative corridor and depth of surgical anatomy. The prognosis for chordoma located at C-1 and C-2 also is poor, with these 2 vertebrae contributing primarily to rotation of the head. Without treatment, tumor progression will result in brainstem, cranial nerve, and spinal cord compression, and progressive instability at the craniocervical junction with intractable pain, with eventual quadriplegia and death. ${ }^{5}$

There were a number of stages to performing this procedure.

\section{Part 1: Posterior Fusion, Oc-C3}

Initial occipitocervical (Oc-C3) fusion was performed (Stryker OASYS) posteriorly for stability, prior to the planned anterior tumor resection. In addition, the posterior fusion was performed so that exact planning of the tumor resection and patient-specific implant reconstruction could be accomplished due to fixed anatomy joining the skull base to the upper cervical spine and to C-3. Neuronavigation was possible because the skull and upper cervical spine were fixed in relative space to provide accuracy during the procedure for navigation.
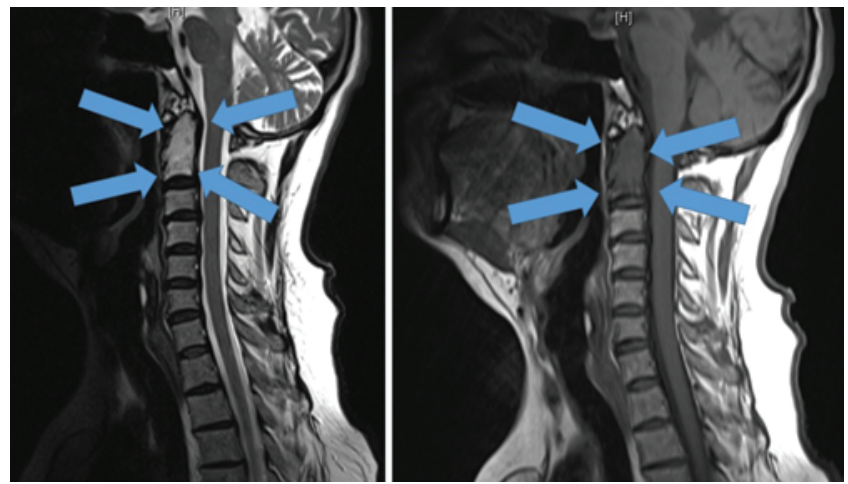

FIG. 1. Left: Sagittal T2-weighted MR image demonstrating chordoma (arrows). Right: Sagittal T1-weighted MR image demonstrating chordoma (arrows). Figure is available in color online only.

\section{Part 2: Implant Design Process}

A transoral anterior approach was planned, which provides an extradural route to the upper cervical spine without brainstem or spinal cord retraction around the craniocervical junction. Given the complex anatomy and challenge of surgical treatment, 3D printing technology was used for surgical planning by constructing an anatomically accurate 3D plastic model of the patient's craniocervical anatomy. The surgeon (R.J.M.) and Anatomics Pty. Ltd. worked together on 3D computer-aided design (CAD) software (Fig. 2A and B) and, using the plastic models, to customize a range of potential implant models to fit the defect after tumor resection. The custom titanium-printed prosthesis was manufactured by Commonwealth Scientific and Industrial Research Organisation (C.S.I.R.O.) (Fig. 2D) based on the resection margins calculated from 3D modeling.

The surgeon correlated the medical imaging data with a 3D plastic model of the patient's spine. A second 3D plastic model incorporating the proposed tumor resection was made. A semitransparent plastic model of the anatomy was also created, with the existing posterior fixation highlighted in red (Fig. 2C). The red screw coloration combined with the semitransparency of the bone in the model confirmed the position of the existing posterior fixation screws with respect to the proposed resection.

Implant design then commenced, using global dimensions implied by the resection and surgeon-provided concept sketches. Plastic implant prototypes were then constructed for a trial fit on the resected anatomy models to test various implant designs (Fig. 2C). These prototypes were inspected by the surgeon. Further implant design refinement was achieved. The fixation screw trajectories were determined by the surgeon using the plastic model and implant prototypes. The fixation screw dimensions
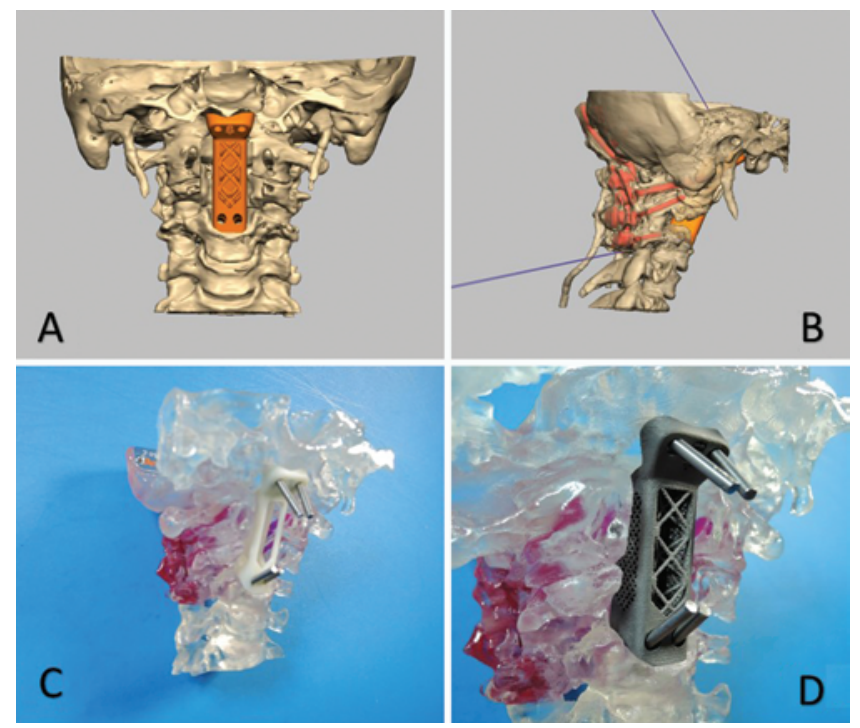

FIG. 2. A: CAD modeling of implant to reconstruct defect from tumor excision. B: Posterolateral view demonstrating prior occipitocervical fusion, and lines demonstrating planned trajectory for screw fixation for the 3D implant. C: Plastic model of implant with planned screw trajectories into clivus and C-3. D: Final model prior to sterilization and surgery. Figure is available in color online only. 
were then calculated in software and reported to the surgeon for prearrangement of implant fixation. The screw trajectories were then built into the implant to aid in screw placement (Fig. 2B-D).

The final implant designs were exported electronically to and built by C.S.I.R.O. The 3D-printed parts proceeded to quality checks. A semitransparent plastic model, implant template, and screw trajectory indicators were supplied and sterilized to assist intraoperatively.

\section{Part 3: Anterior Transoral Approach, Tumor Resection, and 3D Implant Insertion}

General anesthesia was performed via tracheostomy. The patient was positioned supine. The head was fixed in a Mayfield clamp, and a BrainLab reference frame (BrainLab) was attached for neuronavigation. A planned Le Fort midface split was not performed because an adequate approach could be achieved via the transoral corridor. An incision was made in the mucosa of the midline gingiva sulcus to expose the anterior nasal spine. The submucosal nasal floor was dissected along the palate, and the nasal septum was cut and freed from the palatal bone. A midline palate split was performed to allow access to the clivus for the reconstruction phase of the procedure. A linear incision of the posterior oral mucosa was made to allow surgical field access to the clivus, craniocervical junction, and $\mathrm{C}-2$ and $\mathrm{C}-3$ vertebral bodies.

A C-2/C-3 discectomy was performed to identify the inferior extent of the chordoma. To remove the superior extent of the tumor, the anterior $\mathrm{C}-1$ arch was resected and division of the apical and alar ligaments was performed. The lateral borders of the chordoma were identified using CT-based navigation, and en bloc resection of the lesion was achieved.

After the en bloc resection, reconstruction and anterior fixation was completed with the custom-designed 3Dprinted titanium cage filled with bone matrix and 2-screw fixation into the clivus and 2 screws into the C-3 vertebra (Fig. 3). All screw lengths were predetermined based on the preoperative 3D and plastic modeling, and screw angulation was built into the prosthesis to ease screw insertion. Following successful reconstruction, the mucosa and palate split was closed, and the patient was transferred to the intensive care unit. The total length of the procedure was 16.5 hours, with $480 \mathrm{ml}$ of blood loss.

Postoperatively, the patient had temporomandibular joint dysfunction secondary to prolonged stretching of the oral cavity during the procedure. The prolonged operation duration also resulted in an upper esophageal stricture. These complications were attributed to the lengthy period of oral retraction. The patient recovered speech by 8 weeks postoperatively; however, he required percutaneous endoscopic gastrostomy to enable feeding for the initial 3 months following surgery. Radiotherapy targeting the lateral aspect of the tumor resection margin was performed at 6 months postsurgery. There were no complications with this intervention. The patient had normal phonation and swallowing function at his 9-month followup. At the 9-month follow-up, flexion and extension radiographs demonstrated no movement and thus probable fusion.
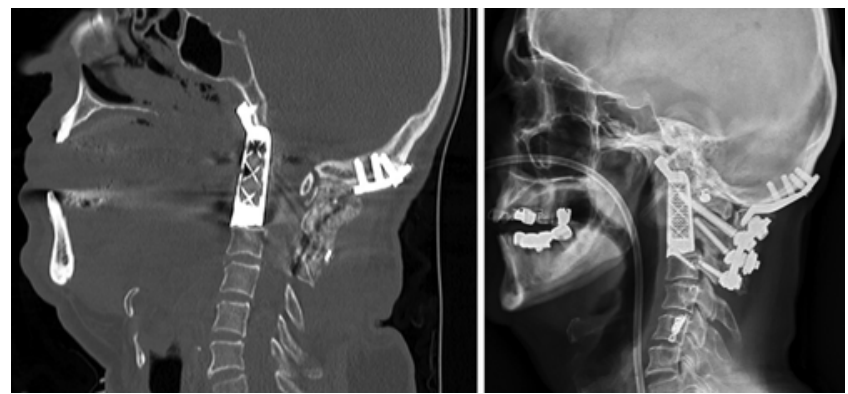

FIG. 3. Postoperative CT (left) and postoperative radiograph (right) demonstrating the 3D-printed titanium implant.

\section{Case 2}

A 52-year-old woman presented with an 18-month history of intractable back pain and unilateral leg pain. The patient was treated conservatively for 2 years with weight loss and physical therapy. Her CT and MRI studies demonstrated an unusual vertebral anomaly, with a congenital hemivertebra at L-5, segmental kyphosis with loss of lordosis, and degenerative changes. The opinion of the surgeon (M.C.) was that a standard fusion performed using a commercially available implant would not provide adequate anatomical restoration and support, based on the patient's unique anatomy.

\section{Operative Approach}

An anterior and posterior fusion approach was planned for the patient. The preoperative CT and MRI studies were used to construct a 3D model of the patient's anatomy as well as to design a customized 3D-printed titanium implant (Fig. 4). A standard L-4/L-5 anterior approach was performed, and the level of pathology was confirmed with radiographs prior to the disc removal, with endplate preparation. The custom 3D-printed titanium implant was packed with the bone graft (Allograft, Ausbiotechnologies) and inserted, with a precision fit. Anterior plate fixation was implemented to secure the motion segment, with additional posterior percutaneous pedicle screws for further stabilization of the L-4/L-5 segment. Radiography was used to confirm correct placement, and antibiotic irrigation was used prior to closure (Fig. 5B and C).

\section{Patient-Specific Implant Design}

The following design criteria were developed for the required patient-specific interbody fusion implant. 1. Al-

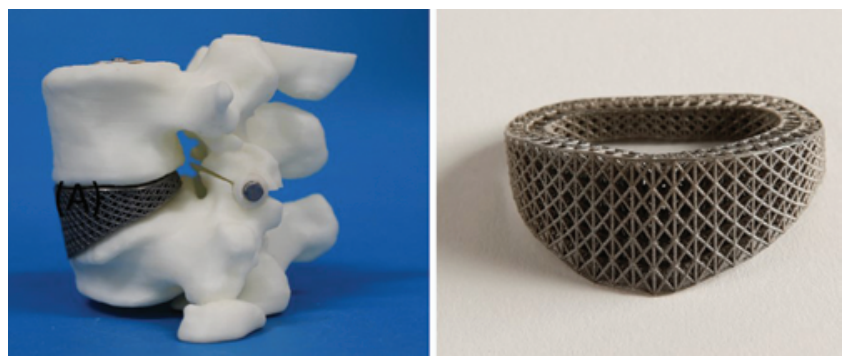

FIG. 4. Left: Plastic model of hemivertebra with planned 3D-printed prosthesis. Right: Prosthesis prior to sterilization and implantation. Figure is available in color online only. 

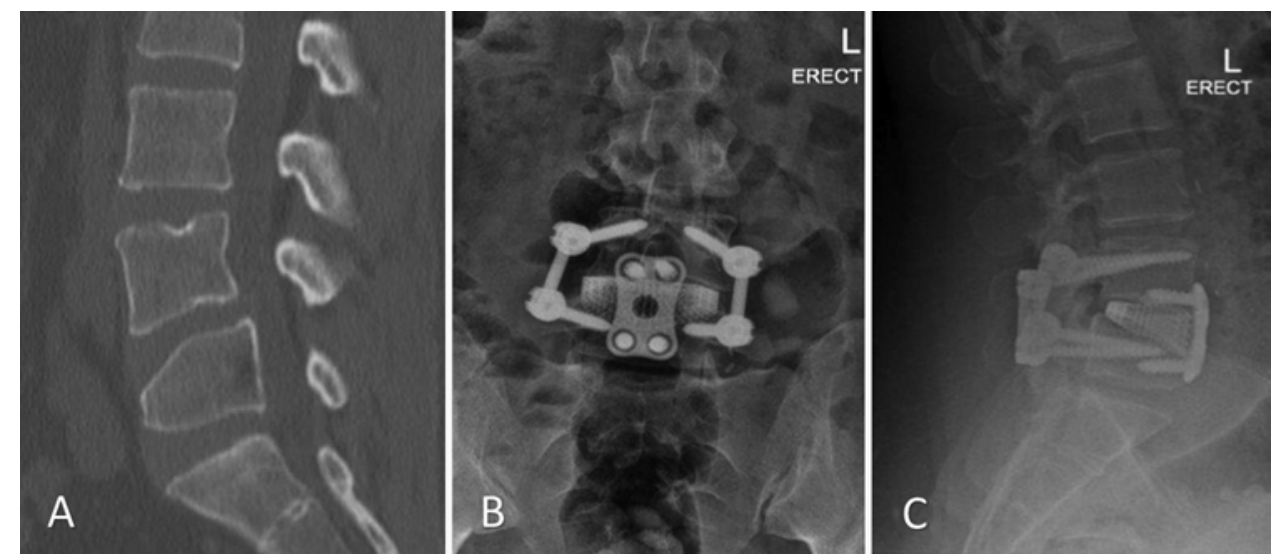

FIG. 5. A: Preoperative midsagittal CT. B: Postoperative anteroposterior radiograph. C: Postoperative lateral radiograph.

low maximum strength to support the weight of the patient while simultaneously providing a large central empty space for bone graft delivery and containment. 2. Ensure minimal support material for 3D printing to reduce postprocessing by being a structure that is "self-supporting" during the building process. 3. Provide maximum sparseness to ensure the best possible postoperative imaging (Fig. 4 right).

The surgeon, in consultation with representatives of the involved 3D implant design and manufacture companies (Anatomics, ProCRO), specified that the implant should effect a lordosis change of $6^{\circ}$. The implant was then designed around this altered geometry to meet the design criteria. The final design was used to manufacture the implant (RMIT University, Advanced Manufacturing Precinct, Carlton, Australia). The 3D-printed parts were then checked for postprocessing as in Case 1 (Fig. 4).
At 12-month follow-up, significant improvements were found in terms of axial back (from preoperative 10 to postoperative 0 ), right leg (from preoperative 2 to postoperative 0 ), and left leg (from preoperative 2 to postoperative 0 ) visual analog scale scores. The Oswestry Disability Index also improved significantly, from $68 \%$ preoperatively to $0 \%$ postoperatively. The 12-month radiological follow-up (Fig. 6) demonstrated solid mature fusion with no failure of fixation and no subsidence.

\section{Discussion}

Treatment of complex spinal pathologies such as a craniocervical junction chordoma or complex spinal congenital deformity requires meticulous surgical technique and considerable preplanning. ${ }^{13,18}$ In the case of the upper cervical chordoma, this particular procedure is associated
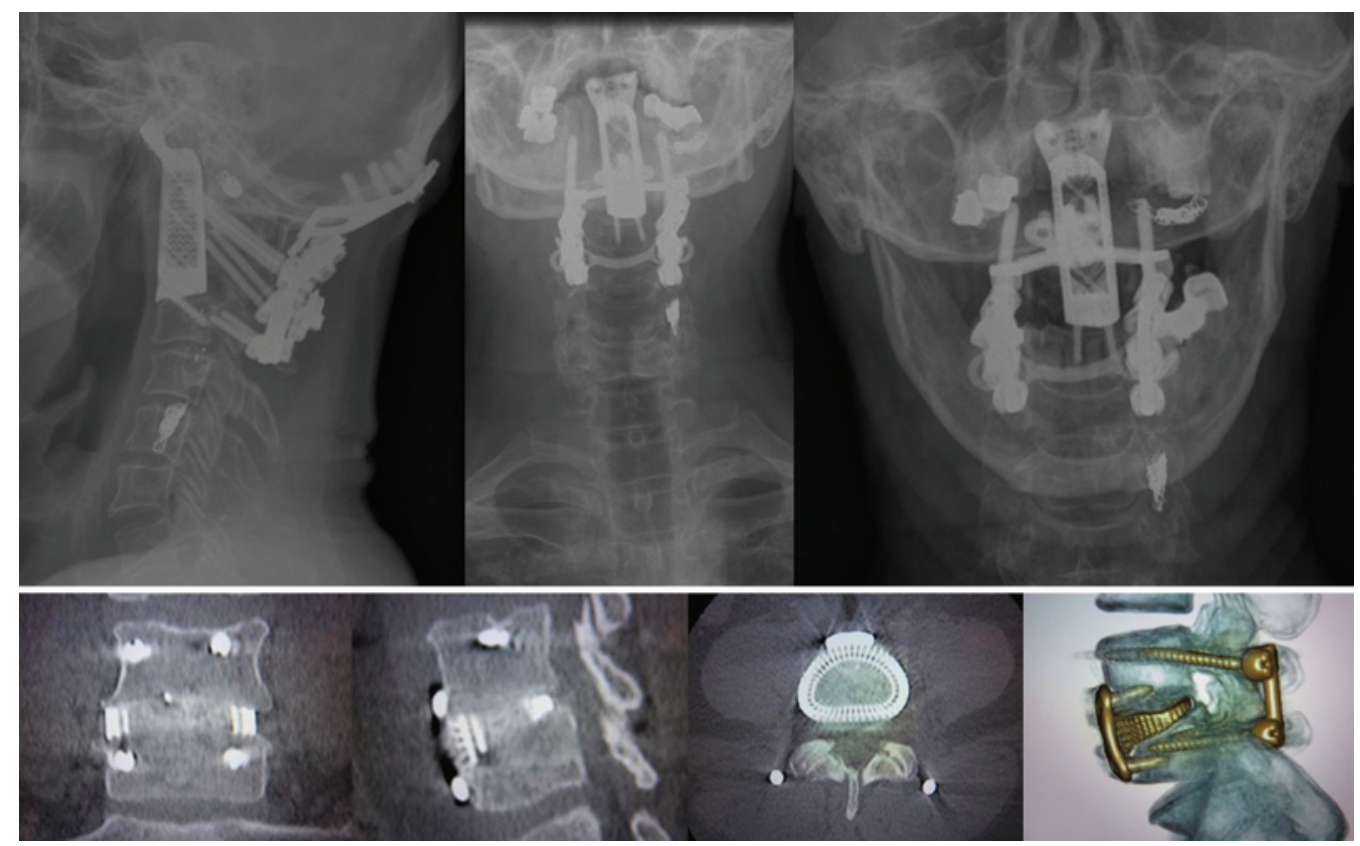

FIG. 6. Upper Row: Case 1. Nine-month follow-up radiographs showing no movement and likely fusion. Lower Row: Case 2. Twelve-month radiographic follow-up demonstrating solid mature fusion, no failure of fixation, and no subsidence. Figure is available in color online only. 
with a high failure and morbidity risk, and such prolonged procedures are particularly high risk in the elderly..$^{10}$ The difficulty and challenge of such complex spinal surgeries can be anticipated and relieved by preoperative planning. The introduction of 3D printing has provided a means of accurately depicting the patient's surgical anatomy, as well as the ability to fashion the custom-designed implants and prostheses that match more closely the unique anatomy of any particular patient and pathology. ${ }^{12-16}$ In both presented cases, the custom-designed and custom-built implants were easily slotted into position, which facilitated the surgery and shortened the operating time, avoiding further complex reconstruction such as the harvesting of rib or fibular grafts and the fashioning of these grafts intraoperatively to fit the defect.

These 2 cases, which we approached by preoperative planning using 3D-printed models as well as custom-designed implants, are part of a larger paradigm of personalized medicine. As an alternative to standard off-the-shelf implants, 3D-printed implants can be customized to the unique anatomy of the patient. Although such developments are exciting, this surgery has not disseminated widely due to the high costs and the requirements of sophisticated software and machinery not easily accessible to the majority of surgeons.

Charles Hull first proposed the 3D printing process in 1986. ${ }^{1}$ Since that time, the 3D printing market has exploded, becoming increasingly more commonplace and lower in cost. ${ }^{9}$ The application of 3D printing in medicine and surgery may offer several benefits, including customization of medical products and implants, improved preoperative planning, reduced operative duration and surgical complexity, and accurate placement of implants and prostheses, as well as enhanced productivity. Particularly in the context of complex spinal and cranial surgery, 3Dprinted neuroanatomical models can be particularly helpful by providing an accurate representation of cranial nerves, vessels, and skull architecture and their relationships, which may be difficult to interpret using standard 2D radiographic imaging. ${ }^{2,8,11,16,17}$ Accurate 3D models can help the surgeon plan and rehearse a safe surgical corridor or approach preoperatively, which may ease the difficulty and lower the operative duration of complex surgical cases.

Although the technique is promising, current barriers include commercial costs of 3D printing of prostheses as well as limited access to high-quality $3 \mathrm{D}$ printers. The proliferation and increasing quality of low-cost desktop 3D printers may be a game changer in the future, and it may facilitate lower-cost, custom-designed implants and prostheses. Several published reports have demonstrated the feasibility of producing patient-specific cranioplasty implants using low-cost desktop 3D printers coupled with cheap and widely available acrylic bone cement. ${ }^{14}$ Possible future directions of research include the combination of 3D-printed implants and biologics, as well as the development of bioceramic composites and custom implants for load-bearing purposes.

\section{References}

1. American Association for the Advancement of Science:
Science and society. Experts warn against bans on 3D printing. Science 342:439, 2013

2. Anderson JR, Thompson WL, Alkattan AK, Diaz O, Klucznik R, Zhang YJ, et al: Three-dimensional printing of anatomically accurate, patient specific intracranial aneurysm models. J Neurointerv Surg 8:517-520, 2016

3. Barker FG: Repairing holes in the head: a history of cranioplasty. Neurosurgery 41:999, 1997 (Letter)

4. D'Urso PS, Askin G, Earwaker JS, Merry GS, Thompson RG, Barker TM, et al: Spinal biomodeling. Spine 24:12471251,1999

5. Harwick RD, Miller AS: Craniocervical chordomas. Am J Surg 138:512-516, 1979

6. Izatt MT, Thorpe PLPJ, Thompson RG, D'Urso PS, Adam CJ, Earwaker JWS, et al: The use of physical biomodelling in complex spinal surgery. Eur Spine J 16:1507-1518, 2007

7. Klein GT, Lu Y, Wang MY: 3D printing and neurosurgeryready for prime time? World Neurosurg 80:233-235, 2013

8. Mao K, Wang Y, Xiao S, Liu Z, Zhang Y, Zhang X, et al: Clinical application of computer-designed polystyrene models in complex severe spinal deformities: a pilot study. Eur Spine J 19:797-802, 2010

9. Martelli N, Serrano C, van den Brink H, Pineau J, Prognon P, Borget I, et al: Advantages and disadvantages of 3-dimensional printing in surgery: A systematic review. Surgery 159:1485-1500, 2016

10. Molina CA, Ames CP, Chou D, Rhines LD, Hsieh PC, Zadnik PL, et al: Outcomes following attempted en bloc resection of cervical chordomas in the $\mathrm{C}-1$ and $\mathrm{C}-2$ region versus the subaxial region: a multiinstitutional experience. J Neurosurg Spine 21:348-356, 2014

11. Namba K, Higaki A, Kaneko N, Mashiko T, Nemoto $S$, Watanabe E: Microcatheter shaping for intracranial aneurysm coiling using the 3 -dimensional printing rapid prototyping technology: preliminary result in the first 10 consecutive cases. World Neurosurg 84:178-186, 2015

12. Schubert C, van Langeveld MC, Donoso LA: Innovations in 3D printing: a 3D overview from optics to organs. Br J Ophthalmol 98:159-161, 2014

13. Song ZL, Feng CK, Chiu FY, Liu CL: The clinical significance of rapid prototyping technique in complex spinal deformity surgery-case sharing and literature review. J Musculoskeletal Disord 4:88-93, 2013

14. Tan ET, Ling JM, Dinesh SK: The feasibility of producing patient-specific acrylic cranioplasty implants with a low-cost 3D printer. J Neurosurg 124:1531-1537, 2016

15. Waran V, Narayanan V, Karuppiah R, Owen SLF, Aziz T: Utility of multimaterial 3D printers in creating models with pathological entities to enhance the training experience of neurosurgeons. J Neurosurg 120:489-492, 2014

16. Weinstock P, Prabhu SP, Flynn K, Orbach DB, Smith E: Optimizing cerebrovascular surgical and endovascular procedures in children via personalized $3 \mathrm{D}$ printing. $\mathbf{J}$ Neurosurg Pediatr 16:1-6, 2015

17. Wurm G, Lehner M, Tomancok B, Kleiser R, Nussbaumer $\mathrm{K}$ : Cerebrovascular biomodeling for aneurysm surgery: simulation-based training by means of rapid prototyping technologies. Surg Innov 18:294-306, 2011

18. Yang JC, Ma XY, Xia H, Wu ZH, Ai FZ, Zhang K, et al: Clinical application of computer-aided design-rapid prototyping in $\mathrm{C} 1-\mathrm{C} 2$ operation techniques for complex atlantoaxial instability. J Spinal Disord Tech 27:E143E150, 2014

\section{Disclosures}

Robert Thompson is an employee of Anatomics Pty. Ltd., who were involved in the design and manufacture of the 3D-printed 
implants reported in this study. Chester E. Sutterlin III is a representative of and has ownership in ProCRO Pty. Ltd., which was involved in the design and manufacture of a 3D-printed implant reported in this study. Dr. Mobbs is a consultant for Stryker, Kasios Biomaterials, and A-Spine Asia.

\section{Author Contributions}

Conception and design: all authors. Acquisition of data: all authors. Analysis and interpretation of data: all authors. Drafting the article: Mobbs, Thompson, Sutterlin, Phan. Critically revising the article: Mobbs, Coughlan, Phan. Reviewed submitted version of manuscript: Mobbs, Coughlan, Phan. Study supervision: Mobbs.

\section{Correspondence}

Ralph J. Mobbs, NeuroSpineClinic, Ste. 7, Level 7 Prince of Wales Private Hospital, Randwick, Sydney NSW 2031, Australia. email: ralphmobbs@hotmail.com. 